\title{
The Effect of Exocrine Pancreatic Function on Chloramphenicol Pharmacokinetics in Patients with Cystic Fibrosis ${ }^{1}$
}

\author{
CHRIS J. DICKINSON, ${ }^{2}$ MICHAEL D. REED, ROBERT C. STERN, STEPHEN C. ARONOFF, \\ TOYOKO S. YAMASHITA, AND JEFFREY L. BLUMER \\ Divisions of Pediatric Pharmacology and Critical Care, Pulmonary Medicine and Infectious Diseases, Rainbow \\ Babies and Childrens Hospital, and Departments of Pediatrics, Pharmacology and Epidemiology and \\ Biostatistics, Case Western Reserve University School of Medicine, Cleveland, Ohio 44106
}

\begin{abstract}
The effect of exocrine pancreatic function on the pharmacokinetics of the choramphenicol oral capsule (CAP-base), chloramphenicol palmitate oral liquid (CAP$\mathrm{P}$ ), and chloramphenicol succinate intravenous (CAP-S) formulations was evaluated in 10 patients, aged 16-30 yr, with cystic fibrosis. Pancreatic insufficiency was assessed in each patient by measuring the absorption of $p$-aminobenzoic acid after oral administration of N-benzoyl-L-tyrosyl-p-aminobenzoic acid which requires chymotrypsin to cleave $p$-aminobenzoic from the parent molecule. In a controlled cross-over design, the overall biodisposition of each formulation was assessed in each patient with or without concurrent administration of oral pancreatic enzymes. The relative amounts of active chloramphenicol available in systemic circulation was CAP-base $>$ CAP-S $>$ CAP-P. Pancreatic enzyme replacement had little effect on the biodisposition parameters for the CAP-base and CAP-S formulation, but significantly increased the peak concentration and bioavailability of the CAP-P formulation. Although pancreatic enzyme replacement improved the absorption characteristics of the CAP-P formulation, absorption remained prolonged and unreliable. Serum concentration-time profiles for either CAP-base or CAP-S consistently exceeded the MIC of important nonpseudomonal pathogens. This finding was not observed after CAP$P$ administration independent of pancreatic enzyme replacement. The results of this study support the continued clinical use of either CAP-base or CAP-S, but the cautious use of CAP-P formulations in CF patients with concurrent pancreatic insufficiency. (Pediatr Res 23: 388-392, 1988)
\end{abstract}

\section{Abbreviations}

CAP, chloramphenicol

CAP-S, CAP-succinate

CAP-P, CAP-palmitate

CAP-base, CAP-free base

iv, intravenous

PER, pancreatic enzyme replacement

CF, cystic fibrosis

N-PABA, N-benzoyl-L-tyrosyl-p-aminobenzoic acid

HPLC, high-performance liquid chromatography

Received September 28, 1987; accepted December 9, 1987

Correspondence and reprint requests Jeffrey L. Blumer, Ph.D., M.D., Chief, Division of Pediatric Pharmacology and Critical Care, Rainbow Babies \& Childrens Hospital, 2101 Adelbert Road, Cleveland, OH 44106.

${ }_{1}^{1}$ Presented at the Society for Pediatric Research (Pediatr Res 18:151A, 1984), May 1984, San Francisco, CA.

${ }^{2}$ Present address Division of Pediatric Gastroenterology, University of Michigan Medical Center, Ann Arbor, MI 48109.
AUC, area under the curve

$\mathrm{Cl}$, clearance

Vdarea, volume of distribution

MIC, minimum inhibitory concentration

CAP is an effective antimicrobial agent widely used in pediatric practice (1). Currently, three systemically available pharmaceutical formulations are available for use in clinical practice: CAP$\mathrm{S}$ for iv administration, CAP-P as an oral suspension, and CAPbase as an oral capsule. Both prodrug formulations (CAP-S and CAP-P) are devoid of antibacterial activity and require hydrolysis in vivo to liberate antimicrobially active CAP. CAP-S appears to be hydrolyzed primarily by hepatic esterases, whereas CAP-P appears to be hydrolyzed within the gastrointestinal tract by pancreatic lipase (1-3). Recent data in young infants and some children have demonstrated erratic hydrolysis of CAP-S, whereas the effects of exocrine pancreatic insufficiency on the hydrolysis of CAP-P remain ill defined $(1,4,5)$.

CAP is commonly used for oral antimicrobial therapy of ambulatory patients with CF. Despite the widespread use of the drug, only limited data are available describing its biodisposition in CF patients. This lack of pharmacologic data appears to result primarily from difficulties inherent in describing the pharmacokinetics of prodrugs. The present investigation had a dual purpose: 1) to describe the overall biodisposition of the three available CAP formulations in patients with $\mathrm{CF}$, and 2) to determine the effects of PER therapy on CAP biodisposition in patients with $\mathrm{CF}$.

\section{MATERIALS AND METHODS}

Subjects. Hospitalized CF patients $\geq 12$ yr old requiring PER who had previously received CAP therapy. were eligible for enrollment into this study. Patients were excluded from enrollment if they had a history of CAP hypersensitivity, leukopenia (white blood cells $<4000$ cells $/ \mathrm{mm}^{3}$ ), thrombocytopenia (platelet count $<100,000 / \mathrm{mm}^{3}$ ), anemia (hematocrit $<35 \%$ ), significant hepatobiliary disease (total bilirubin $>1.5 \mathrm{mg} / \mathrm{dl}$ and/or serum glutamyloxaloacetic transaminase $\geqq 70 \mathrm{IU} /$ liter), renal disease (serum creatinine $>1.5 \mathrm{mg} / \mathrm{dl}$ ), or hypoalbuminemia $(<2.5 \mathrm{~g} /$ dl). Due to interference with the methodology for serum CAP determinations, patients receiving concurrent trimethoprim-sulfamethoxazole, phenobarbital, or drugs known to interfere with CAP biodisposition, were also excluded $(1,6)$. These studies were approved by the Institutional Review Board for Human Subjects Investigation of the University Hospitals of Cleveland, and writ- 
ten informed consent was obtained from each patient and/or family.

Drug administration and sampling. The study was of a controlled cross-over design in which each patient received a single $20 \mathrm{mg} / \mathrm{kg}$ dose, up to a maximum of $1 \mathrm{~g}$, of each of the three CAP formulations. Each dosage form was administered with or without PER for a total of six pharmacokinetic evaluations in each patient. Patients were studied during the morning hours after an overnight fast. Breakfast was withheld though patients were allowed moderate amounts of clear liquids until lunch. When indicated,a single pancreatic enzyme replacement capsule was administered concomitantly with the CAP dose. Patients were allowed lunch with their usual PER a minimum of $3.5 \mathrm{~h}$ after CAP administration. Each pharmacokinetic evaluation was separated by at least $36 \mathrm{~h}$. CAP-S was administered iv through a peripheral vein over $\sim 4 \mathrm{~min}$; CAP-P and CAP-base were administered orally along with $\sim 240 \mathrm{ml}$ water. All three CAP formulations used were obtained from a single manufacturer (ParkeDavis Laboratories, Detroit, MI).

Venous blood samples (minimum $3 \mathrm{ml}$ ) for the determination of CAP were obtained at $0,0.25,0.5,0.75,1,1.5,2,3,5,7$, and $12 \mathrm{~h}$ after iv CAP-S administration and at $0,0.5,1,1.5,2,2.5$, $3,4,5,6,8$, and $12 \mathrm{~h}$ after oral CAP-P and CAP-base administration. Blood was collected in sterile glass tubes, allowed to clot, and immediately centrifuged. Serum was removed and stored at $-70^{\circ} \mathrm{C}$ until analyzed. All samples were analyzed within 14 days of collection.

Assessment of exocrine pancreatic function. Exocrine pancreatic insufficiency was confirmed in each study patient by the use of N-PABA $(7,8)$. Patients discontinued their usual PER therapy for $24 \mathrm{~h}$ and were evaluated after an overnight fast. NPABA, $500 \mathrm{mg}$ in $240 \mathrm{ml}$ orange juice, was administered orally with a large breakfast without PER. Patients were asked to void before N-PABA administration and all urine excreted over the next $6 \mathrm{~h}$ was collected. Urinary PABA was quantitated by HPLC and confirmed colorimetrically by a modification (9) of the procedure originally reported by Bratten and Marshall (10). Urinary PABA excretion $\leqq 55 \%$ of the administered N-PABA dose indicated exocrine pancreatic insufficiency $(8,11)$.

Cleavage of palmitate ester. An in vitro experiment using Pancrease (McNeil Pharmaceuticals, Springhouse, PA) was undertaken to confirm that PER was capable of cleaving the palmitate ester of CAP. CAP-palmitate oral liquid, $200 \mathrm{mg}$, was added to each of three beakers containing $200 \mathrm{ml} 0.45 \%$ sodium chloride and $8 \mathrm{mEq} \mathrm{KCl}$. Beakers were kept in a water bath maintained at $37^{\circ} \mathrm{C}$. The first beaker served as a control and had no Pancrease capsules added. To the second and third beaker, 2 and 4 whole Pancrease capsules were added, respectively. The $\mathrm{pH}$ of the three solutions was decreased to 2.1 with $12 \mathrm{M} \mathrm{HCl}$. After $1 \mathrm{~h}$ the solutions were alkalinized to $\mathrm{pH} 6.5$ with $\mathrm{NaHCO}_{3}$. The solutions were stirred every $15 \mathrm{~min}$. Aliquots $(100 \mu \mathrm{l})$ were withdrawn from each beaker at times 0,1 (just before alkalinization), 2, 3, and $4 \mathrm{~h}$. These were diluted with 900 $\mu \mathrm{l} 0.9 \%$ sodium chloride and assayed immediately for CAP by HPLC as described below. A titrimetric method was used to determine lipase activity at the 4-h sampling (12).

Determination of CAP by HPLC. To prepare serum samples for chromatography, $0.18 \mathrm{ml}$ acetonitrile and $0.02 \mathrm{ml}$ water were added to $0.2 \mathrm{ml}$ serum. The mixture was warmed by hand, vortexed for $10 \mathrm{~s}$, and centrifuged for $2 \mathrm{~min}$. The supernatant was transferred into a $12 \times 75 \mathrm{~mm}$ glass tube which was capped with a polyethylene snap cap and stored in ice until $10 \mathrm{~min}$ before injection.

Chloramphenicol concentrations in serum were determined by HPLC using a Varian model 5020 ternary liquid chromatograph. Briefly, samples were injected into a Valco automated valve fitted with a $25 \mu \mathrm{l}$ loop and pumped at $1.2 \mathrm{ml} / \mathrm{min}$ through a $30 \mathrm{~cm} \times 4 \mathrm{~mm}$ MicroPack MCH $10 \mathrm{C}_{18}$ reverse phase column. The column was temperature controlled at $30^{\circ} \mathrm{C}$ with a column heater and was preceded by a $4 \mathrm{~cm} \times 4 \mathrm{~mm}$ guard column filled with Vydac $40 \mu \mathrm{m}$ pellicular reverse phase packing. Peaks were detected at $276 \mathrm{~nm}$ with a Varian, model UV 100 variable wavelength detector, and printed on a Linear model 291 printer. Peak areas were quantitated on a CDS $111 \mathrm{~L}$ integrator recorder. The mobile phase consisted of acetonitrile: $0.2 \%$ phosphoric acid, 27:73 (v/v). The limit of CAP detectability was $0.2 \mathrm{mg} /$ liter. The between day coefficient of variation was $2.8 \%$ at 25 $\mathrm{mg} /$ liter.

Pharmacokinetic analysis. The biodisposition of CAP after administration of the three different formulations in the presence or absence of PER was characterized for each patient using noncompartmental and graphical techniques (13). Serum CAP concentrations were plotted against time on a semilogarithmic scale. The elimination rate constant, $\beta$, was determined from the slope of the terminal portion of the serum concentration time curve; elimination $t_{1 / 2}$ as $0.693 / \beta$. The area under the serum concentration time curve (AUC) was obtained by using the linear trapezoidal rule up to the final measured serum concentration and extrapolated to infinity. Due to difficulties inherent in determining absolute bioavailability $(\mathrm{F})$ of a compound where the iv preparation is a prodrug, the $\mathrm{F}$ of the CAP-base preparation was assumed to equal $100 \%$ for the purposes of this study. The apparent $F$ of CAP-S and CAP-P, were therefore determined by dividing their respective AUC by each patient's CAP-base AUC. Body $\mathrm{Cl}$ was determined by the formula dose $\mathrm{F} / \mathrm{AUC}_{0}^{\infty}$, whereas the $\mathrm{V}$ darea was determined by $\mathrm{Cl} / \beta$. The peak CAP concentration and time to peak were determined directly from the semilogarithmic plot of each patient's serum concentration time curve. In those instances where the rate of CAP absorption appeared slower than the rate of elimination, the CAP absorption rate constant was determined, and this unusual phenomenon was confirmed by the percent absorbed-time plot (14). Pharmacokinetic parameter estimates dependent on $\beta$ were then analyzed under the conditions of the "flip-flop" phenomenon (15, 16). Statistical evaluation was performed with the paired and unpaired Student's $t$ test and analysis of variance.

\section{RESULTS}

Ten patients (six male) were enrolled into the study. The biodisposition characteristics of CAP-P and CAP-base in the presence or absence of PER were evaluated in all 10 patients, whereas, the biodisposition characteristics of CAP-S were evaluated in eight patients with and 10 patients without PER, respectively. Nine patients used Pancrease as their routine PER therapy and one patient used Coatazyme-S. Patient characteristics are shown in Table 1. Patients ranged in age from 16-30 yr and none demonstrated overt respiratory failure. Urinary PABA re-

Table 1. Patient characteristics

\begin{tabular}{|c|c|c|}
\hline & Mean & Range \\
\hline$n(10)$ & & \\
\hline $\begin{array}{l}\text { Chloramphenicol dose } \\
(\mathrm{mg} / \mathrm{kg})\end{array}$ & 19.5 & $13.6-23.8$ \\
\hline Age (yr) & 22.4 & $16-30$ \\
\hline Wt (kg) & 50.0 & $25-73.7$ \\
\hline Surface area $\left(\mathrm{m}^{2}\right)$ & 1.53 & $1.0-1.9$ \\
\hline Serum creatinine $(\mathrm{mg} / \mathrm{dl})$ & 0.9 & $0.6-1.3$ \\
\hline SGOT (IU/liter) & 33 & $9-60$ \\
\hline Albumin $(\mathrm{g} / \mathrm{dl})$ & 3.5 & $2.8-4.0$ \\
\hline $\mathrm{pCO}_{2}{ }^{*}(\mathrm{~mm} \mathrm{Hg})$ & 37 & $29-44$ \\
\hline Shwachman score $\dagger$ & 52 & $30-78$ \\
\hline Urinary $\mathrm{PABA}(\%) \ddagger$ & 22.4 & $6.4-39.1$ \\
\hline
\end{tabular}

* Partial pressure of carbon dioxide.

$\dagger$ From Ref. 32.

$\ddagger$ PABA excretion as \% N-PABA dose administered (see "Materials and methods"). 
covery was $<40 \%$ in all patients reflecting marked reduction in exocrine pancreatic function.

The ability of Pancrease to cleave the palmitate ester of CAP$P$ in vitro is shown in Figure 1. Similar degrees of palmitate cleavage were observed at the 2-h sampling for both 2 or 4 capsules. At $4 \mathrm{~h}, 49 \%$ of the palmitate ester was cleaved when incubated with 4 Pancrease capsules, compared to $31 \%$ with 2 Pancrease capsules. Lipase activity at $4 \mathrm{~h}$ was $98 \%$ of that initially present.

Figure 2 depicts mean $( \pm \mathrm{SD})$ CAP serum concentration time curves observed after administration of the three different preparations without PER. Peak CAP concentrations were highest after CAP-S administration. They occurred at the first sampling time, 15 min after completion of the iv infusion, and averaged $14.9 \mathrm{mg} /$ liter. Similar concentrations were observed after CAPbase administration. These averaged $13.4 \mathrm{mg} /$ liter but occurred $2.3 \mathrm{~h}$ after oral drug administration. In contrast, the serum concentration time profile observed after CAP-P administration was markedly different from those observed with either the CAP-S or CAP-base preparations. Serum concentrations were substantially lower throughout the 0.25 - to 6-h sampling time for CAP-P. Peak CAP concentrations after CAP-P administra-

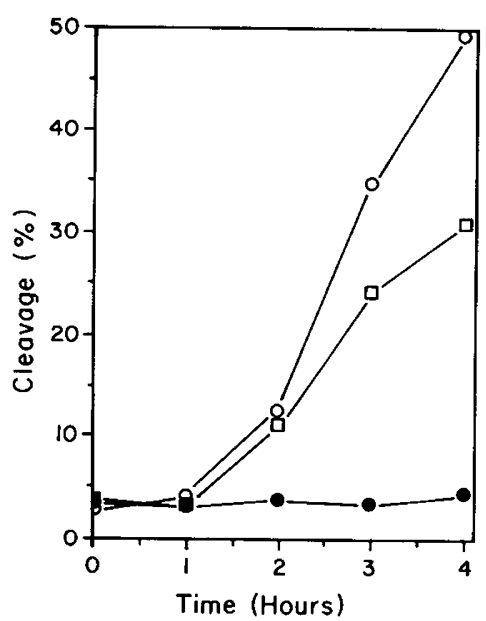

Fig. 1. Palmitate cleavage from CAP-P in vitro. CAP-P was incubated in $200 \mathrm{ml}$ of $0.45 \%$ sodium chloride saline with $8 \mathrm{mEq} \mathrm{KCl}$ to which 0 $(\bullet), 2(\square)$, or $4(O)$ Pancrease capsules were added. After $1 \mathrm{~h}$ solutions were alkalinized and aliquots were withdrawn at $0,1,2,3$, and $4 \mathrm{~h}$ for chloramphenicol determination by HPLC (see "Materials and Methods").

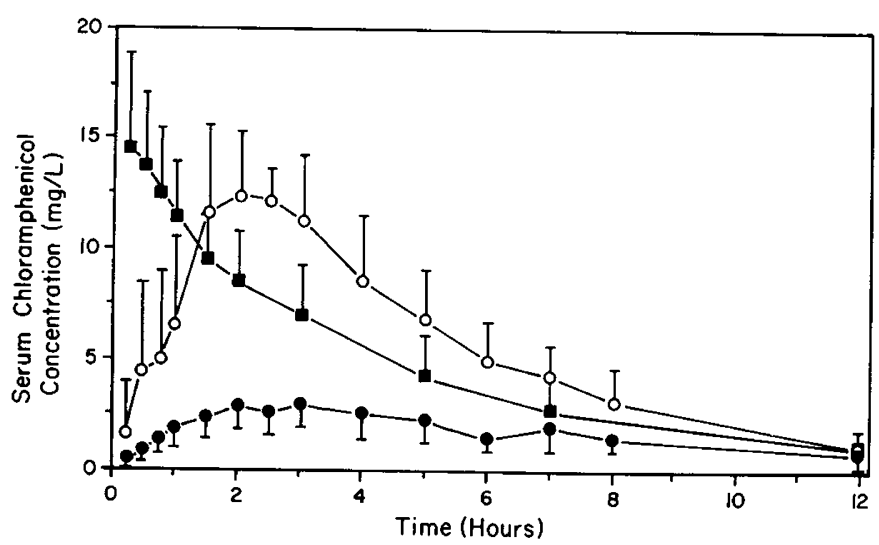

Fig. 2. Chloramphenicol serum concentration-time curve after administration of the three different formulations in the absence of pancreatic enzyme replacement. Numeric plot of mean $( \pm S D)$ serum concentration versus time for chloramphenicol after CAP-S $(n=10)(\square)$, CAP-base, $(n=10)(O)$, and CAP-P $(n=8)(\bullet)$ administration without concurrent exogenous pancreatic enzyme replacement. tion occurred at $2.2 \mathrm{~h}$ averaging $3.2 \mathrm{mg} /$ liter. The CAP-P peak concentration was significantly lower than that observed after CAP-S $(\mathrm{p}<0.001)$ and CAP-base $(p<0.001)$. In addition, time to peak after CAP-P administration was also significantly prolonged as compared to CAP-S $(p<0.001)$ although similar to that observed with CAP-base. In two patients who received CAP$\mathrm{P}$, drug absorption continued throughout the 12-h sampling period. Their data are not included in Figure 2 (see below). Twelve-h trough concentrations were similar for all three CAP preparations ranging from $0.8-1.1 \mathrm{mg} / \mathrm{liter}$.

The pharmacokinetic parameters determined with or without PER for the three different CAP formulations are shown in Table 2. In four patients, two who received CAP-P with PER and two who received CAP-P without PER, CAP continued to be absorbed throughout the 12-h study period. We were unable to perform a pharmacokinetic analysis of CAP-P elimination in these patients, and thus, they are not included in the CAP-P data analysis.

The greatest amount of active CAP in systemic circulation was observed after administration of the CAP-base formulation. In the absence of PER, the CAP AUC after CAP-base was more than the CAP-S AUC and nearly twice as much as the AUC determinations calculated following CAP-P dosing $(p<0.001)$. Although PER did not affect the AUC values for CAP-base, PER significantly increased the AUC after CAP-P administration ( $p$ $<0.05$ ) (Fig. 3). Additionally, the AUC after iv CAP-S was less when infused concomitantly with oral PER than without PER $(p<0.05)$ (Table 2). These AUC differences define the differences in bioavailability observed with CAP-S and CAP-P while using CAP-base as the reference standard. The bioavailability of active CAP for the CAP-S prodrug formulation average $84 \%$ without PER and $69 \%$ with PER, whereas the bioavailability of the CAP-P prodrug formulation without PER averaged $42 \%$ increasing by almost 50 to $64 \%$ with the addition of PER (Table 2).

Substantial variation was observed in the absorption of CAP from the CAP-P preparation, independent of PER. The bioavailability of CAP from CAP-P with PER ranged from 32 to $99 \%$ compared to 19 to $65 \%$ without PER. As mentioned previously in two patients who received CAP-P with and two without PER, drug absorption was incomplete at the end of the 12-h sampling period preventing pharmacokinetic analysis. In contrast, the CAP-S formulation demonstrated consistently higher bioavailability ranging from $52-103 \%$ with and $54-122 \%$ without PER, respectively.

With the exception of AUC, similar pharmacokinetic parameter values were observed for CAP-base and CAP-S formulations (Table 2). The average $t_{1 / 2}$ of CAP ranged from 2.6 to $3.2 \mathrm{~h}$, Vdarea from 1.0 to 1.2 liter $/ \mathrm{kg}$ and total body Cl 4.3 to $4.5 \mathrm{ml} /$ $\mathrm{min} / \mathrm{kg}$. In contrast, the $\mathrm{t}_{1 / 2}$ and Vdarea determinations for CAP$\mathrm{P}$ were significantly different. The CAP-P $t_{1 / 2}$ was significantly prolonged as compared to either CAP-base $(p<0.05$ with PER; $p<0.02$ without PER) or CAP-S ( $p<0.05$ with or without PER), with similar differences observed for Vdarea. These differences, for both the $t_{1 / 2}$ and Vdarea are most likely a result of prolonged absorption of CAP from the CAP-P formulation. However, because the biodisposition of CAP once liberated from the prodrug formulation and entering the vascular compartment should be identical to that observed with CAP administered in any other formulations an alternative pharmacokinetic approach must be applied to the analysis of CAP-P biodisposition. Thus, we also analyzed our CAP-P concentration-time data under the conditions of a "flip-flop" phenomena $(15,16)$. This approach to pharmacokinetic data analysis has been suggested for those situations where a compound's rate of absorption is slower than the elimination rate. These circumstances are suggested by our initial CAP-P results (Table 2). Using this method of data analysis, the CAP-P $t_{1 / 2}$ and Vdarea approached values very similar to those observed after CAP-base and CAP-S administration (Table 3). 
Table 2. Pharmacokinetics of three chloramphenicol preparations in patients with CF [mean (SD)]

\begin{tabular}{|c|c|c|c|c|c|c|}
\hline \multirow[b]{2}{*}{ Parameter } & \multicolumn{2}{|c|}{ CAP-base (capsule) } & \multicolumn{2}{|c|}{ CAP-S (iv solution) } & \multicolumn{2}{|c|}{ CAP-P (liquid) } \\
\hline & $+\operatorname{PER}^{*}(n=10)$ & $-\mathrm{PER} \dagger(n=10)$ & $+\operatorname{PER}(n=8)$ & $-\operatorname{PER}(n=10)$ & $+\operatorname{PER}(n=8)$ & $-\operatorname{PER}(n=8)$ \\
\hline$t_{1 / 2}(h)$ & $2.8(0.7)$ & $2.6(0.7)$ & $2.7(0.8)$ & $3.2(1.2)$ & $5.4(2.0)$ & $5.1(1.9)$ \\
\hline Vdarea (liter/kg) & $1.0(0.3)$ & $1.0(0.2)$ & $1.0(0.3)$ & $1.2(0.5)$ & $2.1(0.8)$ & $2.0(0.7)$ \\
\hline $\mathrm{Cl}(\mathrm{ml} / \mathrm{min} / \mathrm{kg})$ & $4.3(1.1)$ & $4.5(1.1)$ & $4.3(1.2)$ & $4.5(1.1)$ & $4.3(0.9)$ & $4.7(1.2)$ \\
\hline $\operatorname{AUC}(\mu \mathrm{g} \cdot \mathrm{h} / \mathrm{ml})$ & $79.6(23.4)$ & $76.9(22.5)$ & $55.6(18.8)$ & $63.3(21.3)$ & $49.7(19.7)$ & $32.5(15.5)$ \\
\hline $\mathrm{F}(\%)$ & 100 & 100 & $69(18)$ & $84(19)$ & $64(12)$ & $42(16)$ \\
\hline Peak concentration (mg/liter) & $14.7(4.0)$ & $13.4(2.2)$ & $13.9(2.2)$ & $14.9(4.0)$ & $5.4(2.4)$ & $3.2(0.7)$ \\
\hline Time to peak (h) & $1.9(0.7)$ & $2.3(0.7)$ & $0.3(0.1)$ & $0.4(0.1)$ & $3.7(1.4)$ & $2.2(0.7)$ \\
\hline
\end{tabular}

$*+$ PER, with pancreatic enzyme replacement.

$\dagger-\mathrm{PER}$, without pancreatic enzyme replacement.

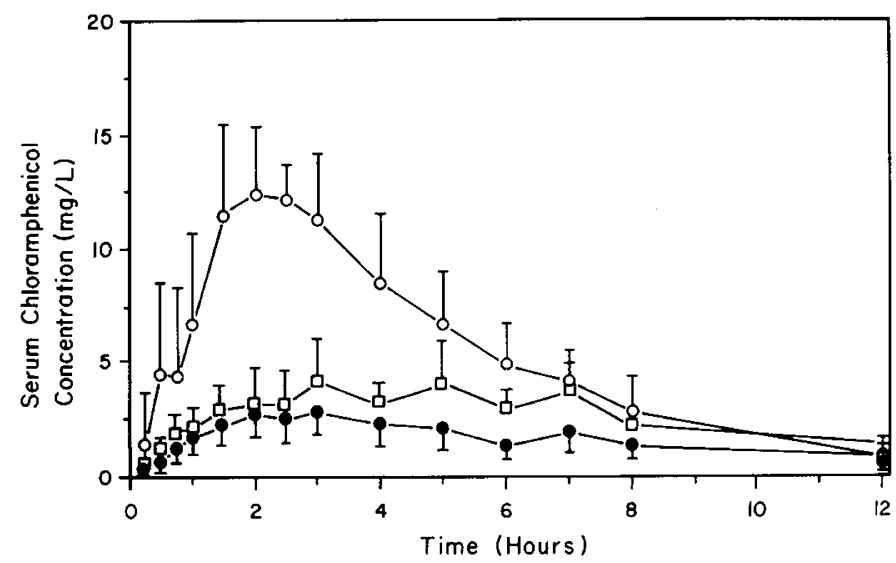

Fig. 3. Effect of concurrent pancreatic enzyme replacement on the biodisposition of CAP.P. Numeric plot of mean ( \pm SD) serum concentration versus time for chloramphenicol after CAP-P $(n=8)(\square)$ with exogenous pancreatic enzyme replacement, and CAP-P $(n=8)(\bullet)$, and CAP-base $(n=10)(O)$ without exogenous pancreatic enzyme replacement.

Table 3. Selected pharmacokinetic parameter estimates after oral administration of CAP-P using two different methods of calculation (mean)

\begin{tabular}{lccccc}
\hline & \multicolumn{2}{c}{$+\operatorname{PER}^{*}(n=8)$} & & \multicolumn{2}{c}{$-\operatorname{PER}(n=8)$} \\
\cline { 2 - 3 } \cline { 5 - 6 } \multicolumn{1}{c}{ Parameter } & $\begin{array}{c}\text { Non- } \\
\text { compartment }\end{array}$ & Flip-flop & & $\begin{array}{c}\text { Non- } \\
\text { compartment }\end{array}$ & Flip-flop \\
\hline $\mathrm{t}_{1 / 2}(\mathrm{~h})$ & 5.5 & 2.7 & & 5.1 & 2.9 \\
Vdarea (liter $/ \mathrm{kg})$ & 2.1 & 1.0 & & 2.0 & 1.1 \\
\hline
\end{tabular}

* See legend for Table 2 .

\section{DISCUSSION}

Patients with $\mathrm{CF}$ frequently receive outpatient antibiotic therapy in an attempt to prolong periods between hospitalization. Although advances in the methods available for ambulatory parenteral drug administration have been realized $(17,18)$, oral antibiotics continue to have widespread use. At the present time, there are three CAP formulations available for the treatment of systemic infection; two of these are prodrugs. Both CAP-S for iv administration and CAP-P the oral liquid preparation, lack antimicrobial activity and require hydrolysis in vivo for activation. In contrast, the capsule preparation, CAP-base contains free CAP in its active form. Despite the long-term availability and use of CAP preparations in CF patients, little pharmacologic data regarding CAP pharmacology is available on this compound for these patients (19-21).

Although early investigators reported therapeutic CAP serum concentrations after CAP-P administration in CF patients (21, 22), Palmer et al. (21) reported low serum concentrations that increased with the coadministration of pancreatic extract. Despite these preliminary data, to our knowledge, no complete pharmacokinetic evaluation of CAP has been performed in $\mathrm{CF}$ patients. Moreover, the frequent occurrence of exocrine pancreatic insufficiency in $\mathrm{CF}$ patients underscores the need to assess the absorption characteristics of the CAP-P preparation, because pancreatic lipases appears important in the liberation of active CAP from this preparation (2).

The pharmacokinetic data generated herein reveal a number of differences in the biodisposition characteristics among the three CAP preparations. The greatest amount of active CAP available in systemic circulation $(\mathrm{F})$, as evidenced by AUC determinations, was observed with the oral CAP-base preparation. Although it is rare that an oral preparation of a drug is more bioavailable than the iv form, this is a direct result of the prodrug nature of the CAP-S iv preparation. Previous studies in non-CF children have reported between 5 and $73 \%$ of an administered CAP-S dose recovered in the urine as the inactive succinate ester demonstrating substantial variability in in vivo hydrolysis $(22,23)$. This variability in the in vivo hydrolysis of the succinate ester most likely explains the differences observed in $\mathrm{F}$ for CAP-S when administered with or without PER. Independent of these differences in drug $\mathrm{F}$ between the CAP-base and CAP-S preparations, both formulations consistently achieved and maintained serum concentrations exceeding the MIC of important nonpseudomonal pathogens. In contrast to the similarities observed in the biodisposition profiles with CAP-base and CAP-S, marked differences were observed with the CAP-P preparation. Up to $6 \mathrm{~h}$ after administration, CAP serum concentrations were substantially less after CAP-P administration as compared with either CAP-S or CAP-base (Figs. 2 and 3). Comparison of absorption characteristics between CAP-base and CAP-P when evaluated without PER revealed a significantly lower peak concentration $(p<0.001)$ for the CAP-P formulation (Table 2; Fig. 2 ). This may partially be explained by the fact that patients with CF have decreased amounts of pancreatic lipase, and therefore cannot consistently hydrolyze CAP-P to CAP for absorption. In addition to these differences, pharmacokinetic analysis was not possible in four patients (two with and two without PER) due to continued absorption throughout the 12 -h study period.

The absorption characteristics observed in our CF patients after CAP-P administration are markedly different from the "adequate" absorption reported by early investigators $(19,20)$ and the biodisposition characteristics described in unaffected infants, children, and adults (24-26). Serum CAP concentrations after CAP-P administration have often exceeded those after iv CAP-S substantiating the use of CAP-P for the treatment of serious pediatric infections $(26,27)$. In contrast, our CAP-P biodisposition data more closely resemble that observed in neonates (28) where incomplete, prolonged, erratic absorption of CAP and poor exocrine pancreatic function (29) has been described. This is consistent with more recent preliminary data in CF patients (21).

The CAP-P $t_{1 / 2}$ and Vdarea data described herein (Table 2) should be interpreted with caution. The comparative pharma- 
cokinetic data for CAP-base and CAP-S or CAP-P from this study and others (25-27) strongly suggest that CAP from the CAP-P preparation continues to be absorbed during the majority of the 12-h study period. This fact is supported by the significantly prolonged CAP-P $t_{1 / 2}$ and Vdarea which are pharmacokinetic parameters heavily dependent on the terminal elimination rate constant $\beta$. Thus, these differences in $t_{1 / 2}$ and Vdarea are most likely artifactual, reflecting the unreliable absorption characteristics of the CAP-P formulation in CF patients. Although $\beta$ is used mathematically to calculate the "tail" of the AUC determination $\left(\mathrm{AUC}_{\tau}^{\infty}\right)$, this parameter estimate is much less dependent on $\beta$ than $t_{1 / 2}$ or Vdarea. However, as a result of the apparently prolonged $\beta$, it is possible that the CAP-P F estimates shown in Table 2 are overestimated. Assessment of the CAP $\mathrm{AUC}_{0}^{r}$ and $\mathrm{AUC}_{0}^{\infty}$ for the CAP-P preparation demonstrates that the AUC $\mathrm{AU}_{0}^{\tau}$ accounted for, on the average, $83.9 \%$ with and $71.2 \%$ without PER, the total $\mathrm{AUC}_{0}^{\infty}$. The decreased influence of $\beta$ on the $\mathrm{AUC}_{0}^{\infty}$ determination is also reflected by the minimal differences observed between the CAP-P, CAP-base, and CAP-S body Cl (Table 2). Moreover, the suggestion of prolonged absorption with the CAP-P formulation is further supported by the relatively equivalent CAP-P $t_{1 / 2}$ and Vdarea parameter estimates obtained when analyzing these data under the conditions of the "flip-flop" phenomena $(15,16)$ (Table 3 ).

The addition of PER significantly increased the $F$ and peak concentration of CAP-P over CAP-P administration without PER (Table 2). The finding of increased absorption of CAP-P when administered with PER was expected because pancreatic lipase is believed to be responsible for cleaving the palmitate ester (2). Glazko et al. (2) first established that pancreatic-biliary secretions were necessary for CAP-P absorption using the rat model, and that a crude bacterial lipase preparation was capable of completely hydrolyzing small amounts of CAP-P. Although PER increased the AUC and peak CAP concentration of the CAP-P preparation, serum concentrations were far below those achieved with CAP-base or CAP-S and may provide inadequate antibacterial activity systemically. It is possible that a greater amount of CAP would have been systemically available from the CAP-P formulation if a larger number of PER capsules would have been administered. This possibility is supported by our in vitro evaluation of palmitate cleavage which showed greater CAP$\mathrm{P}$ hydrolysis when four capsules were used rather than two capsules (Fig. 1). However, CAP was administered after an overnight fast to minimize any potential interference in CAP absorption or PER activity by gastric contents. Furthermore, because exogenous pancreatic enzymes are known intestinal mucosal irritants (30), it appeared prudent to administer only 1 PER capsule during the study period. Clinically, 4 Pancrease capsules was the maximum dose used by any of our study patients during a regular meal, and our in vitro study suggests that as many as 4 Pancrease capsules, containing a total of 16,000 units of lipase, would at best hydrolyze only $50 \%$ of a small amount of CAP-P in $4 \mathrm{~h}$ (Fig. 1). Other investigators have also demonstrated correlations between poor CAP-P absorption and decreased in vitro hydrolysis using different commercial CAP-P preparations (31).

The results of our investigation reveal clinically important pharmacokinetic differences in the biodisposition of CAP in children with CF. Our data support the use of either the CAPbase or CAP-S preparation, when indicated, in the treatment of CF patients. Both formulations showed similar pharmacokinetic parameter estimates and achieved adequate and predictable concentrations in serum which exceeded the MIC of many important nonpseudomonal pathogens. In contrast, markedly different characteristics were observed after administration of the CAP-P formulation. The unpredictable and decreased absorption combined with substantially reduced serum CAP concentrations would recommend against the routine use of the CAP-P formulation in CF patients who require PER. Although PER appeared to improve the overall $F$ of CAP-P, in the presence of the observed variation one would question the reliability of this drug formulation in consistently achieving therapeutic serum and tissue concentrations.

Acknowledgments. The authors thank Tina Capretta, L.P.N., Eloise Lemon, R.N., Carolyn Myers, Ph.D., and Cheryl O'Brien, R.N. for their helpful assistance in the completion of this study.

\section{REFERENCES}

1. Smith AL, Weber A 1983 Pharmacology of chloramphenicol. Pediatr Clin North Am 30:209-236

2. Glazko AJ, Edgerton WH, Dill WA, Lenz WR 1952 Chloromycetin palmitatesynthetic ester of chloromycetin. Antibiot Chemother 2:234-241.

3. Glazko AJ, Dill WA, Kazenko A, Wolf LM, Carnes HE 1958 Physical factors affecting the rate of absorption of chloramphenicol esters. Antibiot Chemother 8:516-527

4. Freidman CA, Lovejoy FC, Smith AL 1979 Chloramphenicol disposition in infants and children. J Pediatr 95:1071-1077

5. Kauffman RE, Miceli JN, Strebel L, Buckley JA, Done AK, Dajani AS 1981 Pharmacokinetics of chloramphenicol and chloramphenicol succinate in infants and children. J Pediatr 98:315-320

6. Powell DA, Nahata MC, Durrell DC, Glazer JP, Hilty MD 1981 Interactions among chloramphenicol phenytoin and phenobarbital in a pediatric patient. J Pediatr 98:1001-1003

7. Gyr K, Stalder GA, Schiffman I, Fehr C, Vonderschmitt D, Fahrlaender H 1976 Oral administration of a chymotrypsin-labile peptide-a new test of exocrine pancreatic function in man (PFT). Gut 17:27-32

8. Nousia-Arvanitakis S, Arvanitakis C, Desai N, Greenberger NJ 1978 Diagnosis of exocrine pancreatic insufficiency in cystic fibrosis by the synthetic peptide N-benzoyl-L-tyrosyl- $p$-aminobenzoic acid. J. Pediatr 92:734-737

9. Smith HW, Finkelstein N, Aliminosa L, Crawford B, Graber M 1945 The renal clearance of substituted hippuric acid derivative and other aromatic amines in dog and man. J Clin Invest 24:388-404

10. Bratton AC, Marshall EK 1939 A new coupling component for sulfanilamide determination. J Biol Chem 128:537-550.

11. Toskes PP 1983 Bentiromide as a test of exocrine pancreatic function in adult patients with pancreatic exocrine insufficiency. Gastroenterology 85:565569

12. Pelot D, Grossman MI 1962 Distribution and fate of pancreatic enzymes in the small intestine of the rat. Am J Physiol 202:285-288

13. Gilbaldi M. Perrier D 1982 Pharmacokinetics, 2nd ed. Marcel Dekker, Inc., New York, pp 409-417

14. Gilbaldi M. Perrier D 1982 Pharmacokinetics, 2nd ed. Marcel Dekker, Inc., New York. pp 149-155

15. Byron PR, Notari RE 1976 Critical analysis of "Flip-Flop" phenomenon in two-compartment pharmacokinetic model. J Pharm Sci 65:1140-1144

16. Ritschel WA 1986 Hardbook of Basic Pharmacokinetics Including Clinical Applications, 3rd ed. Drug Intelligence Publications, Hamilton, pp 298-299

17. Rucker RW, Gunyon HM 1974 Outpatient intravenous medications in the management of cystic fibrosis. Pediatrics 54:358-360

18. Reed MD 1985 Evaluation of antibiotics for home care programs. Drug Intell Clin Pharm 19:288-290

19. Ross S, Burke FG, Rice EC 1952 The use of chloromycetin palmitate in infants and children; a preliminary report. Antibiot Chemother 2:199-207

20. Hodgman J 1961 Chloramphenicol. Pediatr Clin North Am 8:1027-1042

21. Palmer J, Keith H, Huang N 1980 Absorption of chloramphenicol palmitate in patients with cystic fibrosis. CF Club Abstr $21: 82$

22. Sack CM, Koup JR, Opheim KE, Neeley N, Smith AL 1982 Chloramphenicol succinate kinetics in infants and young children. Pediatr Pharmacol 2:93103

23. Kauffman RE, Thirumoorthi MC, Buckley JA, Aravind MK, Dajani AS 1981 Relative bioavailability of intravenous chloramphenicol succinate and oral chloramphenicol palmitate in infants and children. J Pediatr 99:963-967

24. Pickering LK, Hoecker JL, Kramer WG, Kohl S, Cleary TG 1980 Clinical pharmacology of two chloramphenicol preparations in children: sodium succinate (IV) and palmitate (oral) esters. J Pediatr 96:757-761

25. Ambrose PJ 1984 Clinical pharmacokinetics of chloramphenicol and chloramphenicol succinate. Clin Pharmacokinet 9:222-238

26. Yogev R, Kolling WM, Williams T 1981 Pharmacokinetic comparison of intravenous and oral chloramphenicol in patients with Haemophilus influenzae meningitis. Pediatrics 67:656-660

27. Tuomanen EI, Powell KR, Marks MI, Laferriere CI, Altmiller DH, Sack CM, Smith AL 1981 Oral chloramphenicol in the treatment of Haemophilus influenzae meningitis. J Pediatr 99:968-974

28. Shankaran S, Kauffman RE 1984 Use of chloramphenicol palmitate in neonates. J Pediatr 105:113-116

29. Lebenthal E, Lee PC 1980 Development of functional response in human exocrine pancreas. Pediatrics $66: 556-560$

30. Saunders JHB, Wormsley KG 1975 Pancreatic extracts in the treatment of pancreatic exocrine insufficiency. Gut 16:157-162

31. Bell H, Johansen H, Lunde PKM, Andersgaard HA, Finholt P, Midtvedt T, Holum E, Martinussen B, Aarnes ED 1971 Absorption and dissolution characteristics of 14 different oral chloramphenicol preparations tested on healthy human male subjects. Pharmacology 5:108-120

32. Shwachman H, Kulczycki MD 1958 Long-term study of one hundred five patients with cystic fibrosis. Am J Dis Child 96:6-13 